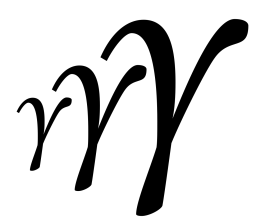

\title{
A Resonance Tracking Approach from Adaptive Control
}

\author{
Meike Gierig $^{a, \star}$ (1) , Lars Flessing ${ }^{b}$ \\ ${ }^{a}$ Leibniz Universität Hannover, Germany \\ ${ }^{b}$ Technische Universität Chemnitz, Germany
}

received 18.07.2018, accepted 01.02.2019, published 15.02.2019

\footnotetext{
* corresponding author: gierig@ikm.uni-hannover.de, +49511 76219059

supervisor: Dominik Kern, TU Chemnitz, Germany
}

\begin{abstract}
Resonance tracking is an approach to measuring concentrations, forces or viscosities. Such vibrationbased measurements appear to be particularly well suited for applications at the micro- or even nano-scale. In order to monitor more than one parameter or parameter ratio simultaneously, a new kind of resonance tracking is developed with methods from adaptive control. It combines parameter estimation methods and state observers to adopt the resonant excitation to vibrating systems with time-varying parameters. At the same time, these parameters are measured. This approach is exemplified at two single-input single-output (SISO) systems: a linear spring-mass-damper oscillator and a weakly nonlinear oscillator of Duffing-type.
\end{abstract}

Keywords: vibration-based measurement, resonance tracking, adaptive control, parameter estimation

\section{Introduction}

If we consider a guitar string that is stretched, we know its pitch will be higher than in the unstretched condition. Therefore we should be able to conclude the tone of the string from its stretch. Varying system parameters, such as mass, damping, stiffness or functional expressions of them influence the vibrations and therefore enable vibration-based measurements. Typical applica- tions for this principle are fault detection [13], oscillating rheometers [5] or tissue differentiation [9]. Microelectromechanic systems (MEMS) are an emerging field where this principle is applied to gyroscopes [15], concentration measurements [8], identification of biomarkers [22] and as an enabler for quantum dot microscopy [23]. In most of these measurement systems, the natural frequencies are either identified via frequency sweeps and subsequent spectrum analysis or via resonance tracking, whereas the latter is faster and enables continuous measurements. The progress in electronics advances the application of new concepts for resonance tracking in vibration-based measurements. By adaptive control, we mean to measure the system parameters via estimation algorithms and to reconstruct all the system states with an observer in order to adaptively tune the controller to track resonance. The measurement itself does not necessarily depend on resonant excitation, however near resonance high amplitudes provide reasonable sensor signals regarding the signal-to-noise ratio. This new measurement concept for resonant vibrations differs from conventional ones, such as autoresonance [2] and phase control [6, 7, 18]. The latter track the resonance by its characteristic phase shift or magnitude peak, from which the measured parameters can be derived. The new approach, employing adaptive 


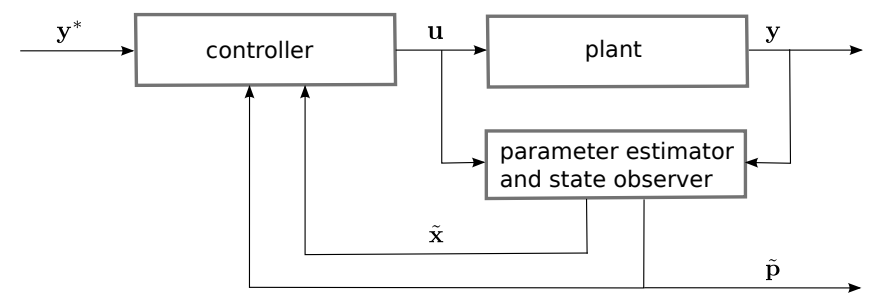

Figure 1 - The control system consists of a plant (states $\mathbf{x}$, time-varying parameters $\mathbf{p}$ ), a tunable controller, and a compound of parameter estimator and state observer

control, appears to be more robust and provides additional information at the expense of computational cost Due to advances in the processing power of current microprocessors, the implementation of more complex algorithms in hand-held measurement systems is now possible. In that regard, field programmable gate arrays (FPGA) offer fast computation for industrial use at a moderate price. The primary application field of resonance tracking is vibration-based measurements, which will serve as motivation in this paper, though this principle is not only limited to measuring. Resonance tracking is also relevant for running actuators at high amplitudes, e.g., ultrasonic generation in machine tools [21]. Besides tuning the excitation to the system, it is also relevant to tune the system properties to the excitation frequency, e.g. in energy harvesting [12].

We begin in Section 2 by the definition of the control task. Its solution follows in Section 3 and Section 4 for a linear oscillator and a weakly nonlinear oscillator of Duffing type, respectively. Some naturally arising questions about limitations of this approach, as well as generalizations to nonlinear and multiple-input multipleoutput (MIMO) systems are addressed in Section 5.

\section{Control Task}

As a preliminary, the notions of measure and sense in this paper are defined as follows. Measurement means the estimation of system parameters influencing the vibration. This is the output of the control system and ultimately its purpose. Whereas sensing refers to the operation of the sensor inside the control loop, meaning the signal path from the system output to the compound of parameter estimator and state estimator.

The control structure is depicted in Figure 1. The input signal $\mathbf{u}$ is the excitation. The excitation frequency is controlled to match one of the plants eigenfrequencies, which change over time. The goal of the controller is the adjustment of the plant input $\mathbf{u}$ in order to keep the system oscillating near resonance or at some spe- cific frequency ratio, while the system parameters are time-varying and thus the eigenfrequencies too. The calculation of the matching excitation frequency is feasible, once the system parameters and its states are known. Consequently, it is reasonable to combine a parameter estimation algorithm, a state observer, and a tunable controller. The parameter estimator receives the input signal $\mathbf{u}$ and the output signal $\mathbf{y}$, of which estimations of specific system parameters are calculated. Both signals $\mathbf{u}, \mathbf{y}$ are also received by the state observer, which processes them together with the plant model, incorporating the parameter updates from the estimator. Following the concept of explicit adaptive control [10], the estimated parameters $\tilde{\mathbf{p}}$ and the observed state vector $\tilde{\mathbf{x}}$ are then fed into the controller that calculates the matching excitation.

\section{Linear Oscillator}

We illustrate our approach to solve the control task for the classical linear oscillator with one degree-of-freedom

$$
m(t) \ddot{x}(t)+d(t) \dot{x}(t)+c(t) x(t)=u(t),
$$

which contains three physical parameters: $m, d$, and $c$. Regarding mechanics, these parameters correspond to mass, damping, and stiffness, whereas $x$ denotes displacement and $u$ the applied excitation force. Concerning electric circuits, the parameters correspond to inductance, resistance, and the inverse capacity in a series resonance circuit, whereas $x(t)$ stands for electric charge and $u(t)$ for the input voltage. Some or all of the three parameters may vary with time, and we measure them by sensing only some states. In our examples we assume the position/charge $x(t)$ known and the velocity/current $\dot{x}(t)$ unknown.

\subsection{Resonant Excitation}

The sensitivity of a sensor or the efficiency of an actuator can be increased in many applications by an increase of the oscillation amplitudes. To ensure this, the controller calculates the matching, typically resonant excitation from the current states, while it adapts to changing parameters. The output of the controller is a harmonic excitation, here formulated as real part of the complexvalued exponential function

$$
u(t)=\mathfrak{R e}\left\{\hat{f} \mathrm{e}^{j\left(\Omega t+\varphi_{f}\right)}\right\},
$$

with imaginary unit $j^{2}=-1$, force amplitude $\hat{f} \in \mathbb{R}_{+}$, radian frequency $\Omega \in \mathbb{R}_{+}$and phase shift $\varphi_{f} \in \mathbb{R}$ and $0 \leq \varphi_{f}<2 \pi$. These parameters are determined so that 
the system oscillates without transience in a steadystate. Generally, the response consists of a solution of the homogeneous equation and a particular solution

$$
x(t)=x_{h}(t)+x_{p}(t) .
$$

The transient effect is described by the solution of the homogeneous equation, while the particular solution describes a harmonic oscillation with the angular frequency identical to the excitation frequency

$$
x_{p}(t)=\mathfrak{R e}\left\{\hat{x} \mathrm{e}^{j\left(\Omega t+\varphi_{x}\right)}\right\},
$$

with amplitude $\hat{x} \in \mathbb{R}_{+}$and phase shift $\varphi_{x} \in \mathbb{R}$. For an oscillation in steady-state without transient $\left(x_{h}=0\right)$, the particular solution has to satisfy the initial conditions

$$
\begin{aligned}
& x\left(t_{0}\right)=x_{p}\left(t_{0}\right)=x_{0}, \\
& \dot{x}\left(t_{0}\right)=\dot{x}_{p}\left(t_{0}\right)=v_{0} .
\end{aligned}
$$

For general resonance tracking a certain frequency ratio $\eta=\frac{\Omega}{\omega_{0}}$ is desired. Hereby, $\omega_{0}$ denotes the eigenfrequency of the system. In this paper we consider exact resonant excitation, i.e. $\eta=1$, implying

$$
\Omega=\omega_{0}=\sqrt{\frac{c}{m}} .
$$

The amplitude and phase are related with the initial conditions

$$
\begin{gathered}
\hat{x}=\sqrt{x_{0}^{2}+\frac{v_{0}^{2}}{\omega_{0}^{2}}}, \\
\varphi_{x}=\arctan \left(\frac{-v_{0}}{\omega_{0} x_{0}}\right)
\end{gathered}
$$

and determined at the beginning or update time $t_{0}$. A state observer is required here since typically either position or velocity is sensed. Thus, the observer is used to reconstruct the missing state. The matching excitation follows directly from inserting $x_{p}$ into equation (1)

$$
u(t)=\mathfrak{R e}\left\{\left(c-m \omega_{0}^{2}+j d \omega_{0}\right) \hat{x} \mathrm{e}^{j\left(\omega_{0} t+\varphi_{x}\right)}\right\} .
$$

This procedure keeps both, excitation $u$ and response $x$ harmonic and monofrequent. In summary, the obtained control law gives the matching excitation, provided that the system parameters and the initial state are known, which will be explained hereafter.

\subsection{Parameter Estimation}

The parameter estimator should provide good approximations for the parameters $m, d$, and $c$ of the oscillating system after each time step. Throughout this section, we assume that the parameter functions $m, d$, and $c$ are piecewise constant. Therefore we can represent the equation of the linear oscillator either in the time domain or in the frequency domain with constant parameters

$$
\begin{aligned}
\ddot{x}(t)+2 \delta \dot{x}(t)+\omega_{0}^{2} x(t) & =\frac{1}{m} u(t), \\
X(s) s^{2}+2 \delta X(s) s+\omega_{0}^{2} X(s) & =\frac{1}{m} U(s),
\end{aligned}
$$

with $2 \delta=\frac{d}{m}$ and $\omega_{0}^{2}=\frac{c}{m}$. Otherwise, we would have to calculate the Laplace-transform of the yet unknown parameter functions. This assumption limits the validity of the measurements to either slow or infrequent parameter changes in comparison to the measurement time, i.e., the time points which are evaluated to obtain a parameter estimation. Further, we assume that the position is sensed with a constant sampling rate $\frac{1}{\Delta t}$. Consequently, the parameters of the time-continuous representation, equation (3b), cannot be obtained directly, but only in a discrete sense from the difference equation on the time grid $t_{k}=k \Delta t$

$$
\begin{array}{r}
x\left(t_{k-2}\right)+a_{1} x\left(t_{k-1}\right)+a_{0} x\left(t_{k}\right) \\
=b_{2} u\left(t_{k-2}\right)+b_{1} u\left(t_{k-1}\right), \\
X(z) z^{-2}+a_{1} X(z) z^{-1}+a_{0} X(z) \\
=b_{2} U(z) z^{-2}+b_{1} U(z) z^{-1},
\end{array}
$$

where (4b) is the Z-transform of (4a). We also assume that the initial condition for the Laplace transform of the system is zero. We further assume a standard model for analog-to-digital conversion (ADC), which holds each sample value constant for the sample interval. With this zero-order hold, the following correspondence is found from a table of Laplace- and Z-transform pairs [19]

$$
\begin{aligned}
& a_{1}=-2 E C, \\
& a_{0}=E^{2}, \\
& b_{2}=\frac{E}{m \omega_{0}^{2}}\left(\delta S-\omega_{d} C+E^{-1}\right), \\
& b_{1}=\frac{E}{m \omega_{0}^{2}}\left(E-\frac{\delta}{\omega_{d}} S-C\right),
\end{aligned}
$$

where $\omega_{d}=\sqrt{w_{0}^{2}-\delta^{2}}$ denotes the damped radian eigenfrequency, and parameters $E=\exp (\delta \Delta t), C=\cos \left(\omega_{d} \Delta t\right)$, and $S=\sin \left(\omega_{d} \Delta t\right)$. In order to determine the four unknown parameters $a_{1}, a_{0}, b_{2}$, and $b_{1}$, at least four difference equations (4a), evaluating $x\left(t_{k-5}\right), \ldots, x\left(t_{k}\right)$ and $u\left(t_{k-5}\right), \ldots, u\left(t_{k-1}\right)$, are necessary. As the resulting linear system of equations may become ill-conditioned, additional equations with position and excitation values from previous time points are included. On the one 
hand, if the parameters change, then including difference equations (4a) that correspond to old parameter values impact the correct estimation of the parameters, and thus we expect a good approximation of new parameters only after a sufficient number of time steps. On the other hand, the measurement is stabilized by including more equations. A good compromise was found by fading memory, where previous values are weighted less than newer ones. In detail, the $n$th equation is multiplied by a scalar weighting factor $r^{n}$, where $0<r<1$. For a given tolerance $\tau>0$, let $N \in \mathbb{N}$ be the smallest number such that $r^{N}<\tau$ and $N>4$. Then we solve the overdetermined system

$$
\begin{aligned}
& r^{n}\left(x\left(t_{k-2-n}\right)+a_{1} x\left(t_{k-1-n}\right)+a_{0} x\left(t_{k-n}\right)\right) \\
& \quad=r^{n}\left(b_{2} u\left(t_{k-2-n}\right)+b_{1} u\left(t_{k-1-n}\right)\right) \quad n=0, \ldots, N
\end{aligned}
$$

with the method of least squares for $a_{1}, a_{0}, b_{2}, b_{1}$ and convert them to the desired parameter estimates $\tilde{m}, \tilde{d}$, and $\tilde{c}$. For generality and ease of implementation, we do not rely on analytical expressions, such as (5), but use an approximation instead. Following Tustin [17], the conversion between discrete-time and continuoustime model is approximated by insertion of the bilinear transformation

$$
z=\frac{2+s \Delta t}{2-s \Delta t}, \quad s=\frac{2}{\Delta t} \frac{z-1}{z+1}
$$

into equation (4b) or (3b), respectively. Here is assumed that the sampling frequency $\frac{1}{\Delta t}$ is much faster than the sensed vibrations, otherwise, prewarping would improve the approximative conversion.

Note, that old values still enter the estimations, so a parameter change cannot be measured immediately.

\subsection{State Observer}

The objective of the observer is to reconstruct unsensed states of the system. In the present case the position is sensed and the velocity needs to be estimated. Both states enter equation (2) whereof the input for the controller is calculated. A powerful observer of simple structure is the one by Luenberger [11]. This observer suites the goal better than a Kalman filter, as it ensures a determined convergence of the states as well as a very easy implementation. In principle, the Luenberger observer uses a feedback of the difference between the sensed state $x$ and its observed value $\tilde{x}$

$$
\begin{aligned}
& \dot{\tilde{x}}(t)=\tilde{v}(t)+s_{1}(x(t)-\tilde{x}(t)), \\
& \dot{\tilde{v}}(t)=\frac{u(t)}{\tilde{m}}-\frac{\tilde{d}}{\tilde{m}} \tilde{v}(t)-\frac{\tilde{c}}{\tilde{m}} \tilde{x}(t)+s_{2}(x(t)-\tilde{x}(t))
\end{aligned}
$$

to reconstruct the hidden state, the velocity. The dynamics of the Luenberger observer are adjusted by its free

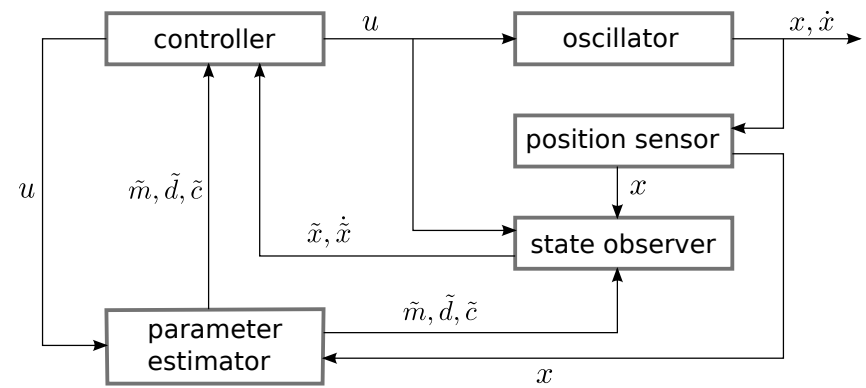

Figure 2-Control structure for resonance tracking of the linear oscillator

parameters $s_{1}$ and $s_{2}$. A reasonable choice is the placement of the observer poles at the aperiodic limit with a time constant greater, i.e. faster, than that of the plant.

\subsection{Example}

The concepts specified in the previous sections are applied to a virtual rheometer, which is simplified to a spring-mass-damper system. Its parameters are summarized in Table 1.

According to the specific control structure in Figure 2, the oscillator receives its input from the controller, and a position sensor samples its output. While the parameters of the oscillator are estimated, its velocity is approximated using the state observer. Note, that the state observer depends on the parameter estimator but not vice versa. In the previous sections, each component was analyzed separately assuming ideal or isolated inand outputs. Since the complete control loop with all interactions is challenging to analyze rigorously, numerical simulations are performed in order to assess the stability and dynamics of the measurements. As a worst case scenario, simulations with step changes of the parameters $m, d$, and $c$ at quarter, half and three-quarter of the simulation time are conducted. The results depicted in Figure 3 show that the parameter estimator overshoots shortly after a parameter change. This effect occurs not only in the changing parameter itself but also in the others since the previous measurements still have a remarkable impact on the calculation. After $0.2 \mathrm{~ms}$ (less than an oscillation period) the estimated parameters match the actual ones with a relative error less than $1 \%$. This settling time coincides with the measurement time, i.e., the time points evaluated for the parameter estimation. In terms of fading memory $\left(r^{0}=1, r^{1}=0.8\right.$, $\ldots, r^{49} \approx 1.8 \times 10^{-5}$ ) the oldest points entering the measurement seem to have no impact, however, they are still active and prevent an ill-conditioning of the system of equations. Although the parameter estimation is fast, it has to be ensured that the peaks, occurring after the parameter change, do not destabilize the control loop. 
Table 1 - Model and simulation parameters of the linear oscillator

\begin{tabular}{llllll}
\hline variable description & variable & value & variable description & variable & value \\
\hline initial position & $x_{0}$ & $0.02 \mathrm{~m}$ & simulation time & $t_{\text {sim }}$ & $3.6 \times 10^{-3} \mathrm{~s}$ \\
initial velocity & $\dot{x}_{0}$ & $0 \mathrm{~m} / \mathrm{s}$ & time step & $\Delta t$ & $3.6 \times 10^{-6} \mathrm{~s}$ \\
mass & $m$ & $(80-120) \times 10^{-9} \mathrm{~kg}$ & fading memory factor & $r$ & 0.8 \\
spring stiffness & $c$ & $(25-35) \mathrm{N} / \mathrm{m}$ & time points per measurement & $M$ & 50 \\
viscous damping & $d$ & $(1.91-4.04) \times 10^{-5} \mathrm{Ns} / \mathrm{m}$ & total time steps & $N$ & 1000 \\
undamped radian eigenfrequency & $\omega_{0}$ & $(1.44-2.09) \times 10^{4} \mathrm{rad} / \mathrm{s}$ & excitation update interval & $N_{\text {exc }}$ & 50 \\
frequency ratio & $\eta$ & 1 & observer dynamics & $\delta_{\text {obs }}$ & $10 \delta$ \\
\hline
\end{tabular}
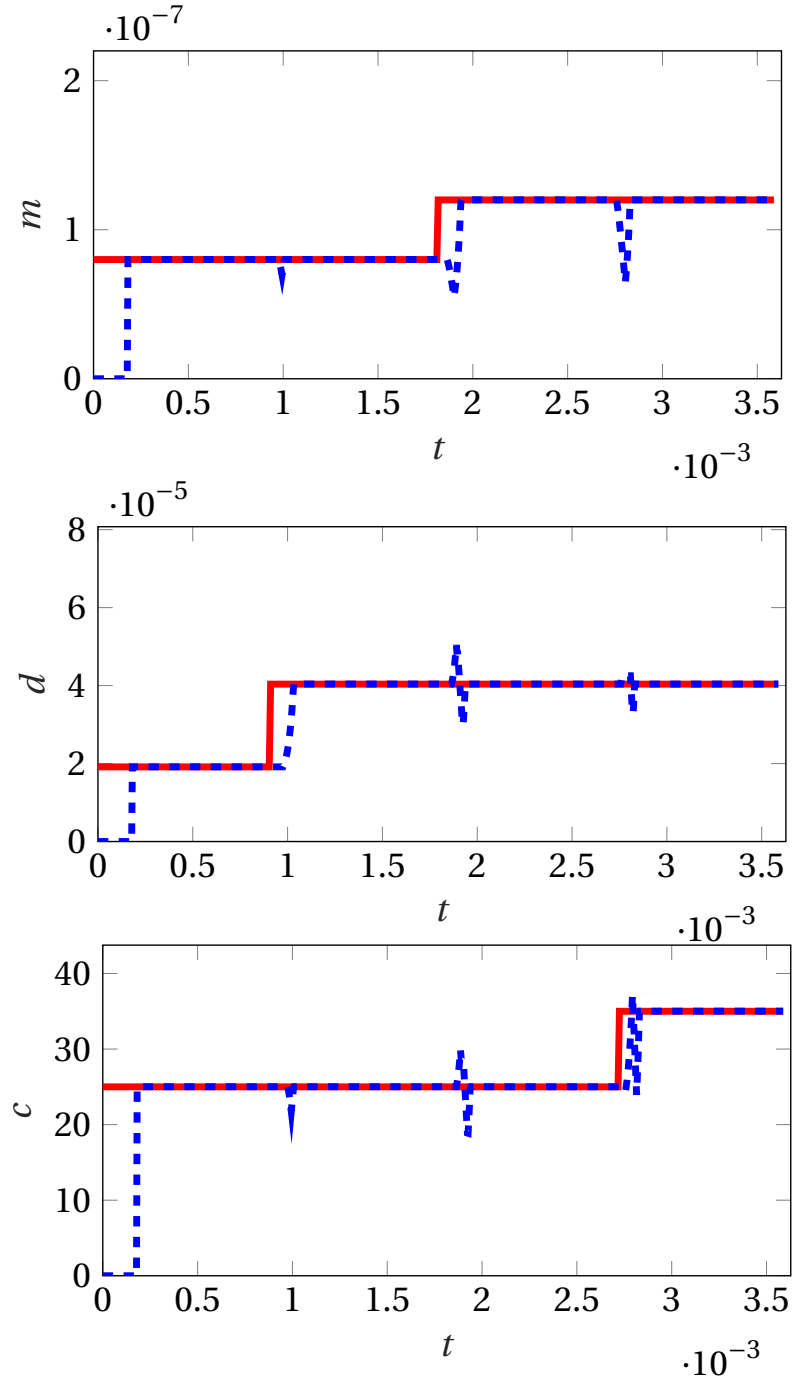

Figure 3 - Real (solid red lines) and estimated (dashed blue lines) parameters of the linear oscillator

For this reason, additional safety-mechanisms are included. First, a Chebyshev low-pass filter [14] is used to smooth the overshoot of the measured parameters. Additionally, it is monitored, if the corresponding energy in the system is consistent. If the fluctuations of energy are too high, the controller ignores the current estimations and continues the excitation based on previous values of the system parameters. Altogether, the safety-

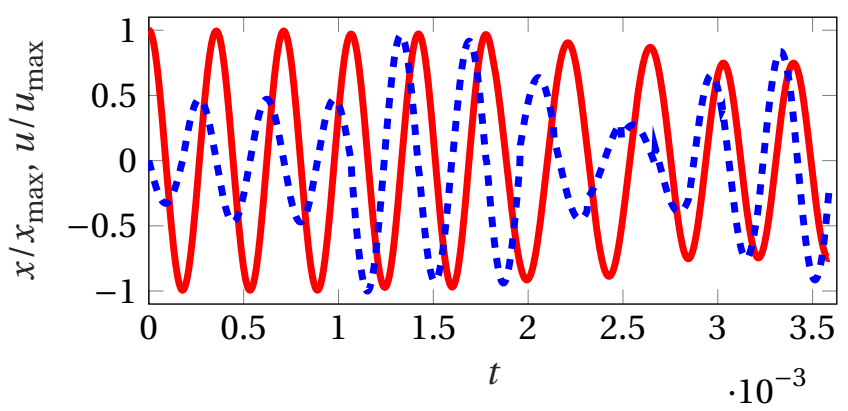

Figure 4 - Position $x$ (solid red line) and excitation $u$ (dashed blue line) of the linear oscillator

mechanisms allow steady measurements and keep the system continuously in resonance without causing too high amplitudes, as depicted in Figure 4. The time scales of the reaction time and the oscillation are close to each other. Additionally, the approach is robust under the limitations of short but large overshoots of the estimated parameters. The stationary deviation of these parameters is less than $1 \%$.

\section{Modified Duffing Oscillator}

A mechanical spring-mass-damper system with a quadratic and a cubic nonlinearity is used as an example in this section. In addition to the classical Duffing oscillator, it contains a quadratic term and hence is referred to as modified Duffing oscillator. Its equation of motion is given by

$$
\begin{aligned}
\ddot{x}(t)+2 D \dot{x}(t) & +x(t) \\
& +\varepsilon\left(a_{2}(t) x^{2}(t)+a_{3}(t) x^{3}(t)\right)=u(t),
\end{aligned}
$$

where $D$ denotes the damping ratio and $0<\epsilon \ll 1$ ensures that the nonlinear term influences the equation only weakly. This restriction leads to an oscillatory solution that is similar to the solution of the linear system that is obtained by setting $\epsilon=0$. As a consequence, this allows us to use the state observer introduced in section Section 3.3 also for the weakly nonlinear system (6). The parameters $a_{2}$ and $a_{3}$ of the nonlinear terms are chosen 


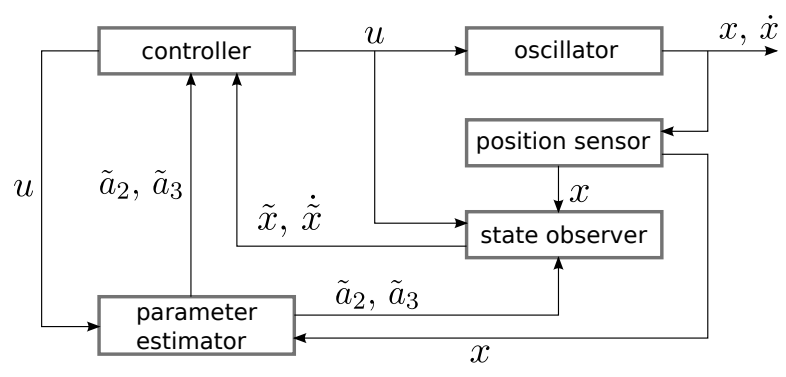

Figure 5 - Control structure for resonance tracking of the modified Duffing oscillator

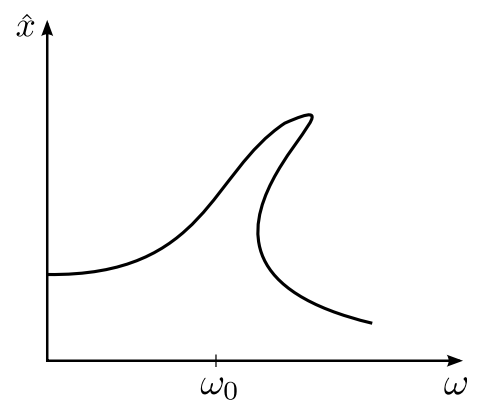

Figure 6 - Exemplaric magnitude response of a weakly nonlinear system

as measurement variables, i.e., they are time-varying and need to be estimated, while the other parameters $D$ and $\varepsilon$ are known and remain constant. As in the previous example, only the position $x$ is assumed to be available as signal and thus the second state variable $v(t)=\dot{x}(t)$ needs to be observed. Although the control loop, shown in Figure 5, shares the same structure with the loop for the linear case, the implementation of its elements (controller, parameter estimator and state observer) is different.

\subsection{Excitation Near Resonance}

Concerning forced oscillations in nonlinear systems, often the frequency-response relations are distorted in comparison to the linear ones. An example is shown in Figure 6, where the magnitude response is depicted. Depending on the plant, the magnitude response bends to lower or higher frequencies. The former is called softening effect, the latter hardening effect. Near the maximal amplitude, up to three amplitude values may relate to a single frequency value. As a consequence, step changes in the steady-state amplitude may occur, and the maximum amplitude may only be reached by a frequency up or down sweep. To overcome this issue the eigenfrequency of the linear part, here $\Omega=\omega_{0}=1$, is tracked similarly to linear systems. Again, the simulation time is subdivided by the update times, thus at each update time, we speak of an initial state. If the desired motion of the oscillator (plant output) is harmonic, then its initial state $x_{0}, v_{0}$ can be cast into a complex representation of magnitude $\hat{x}$ and modulus $\varphi_{x}$ by equation (2). Thus the desired harmonic oscillation $x(t)=\mathfrak{R e}\left\{\hat{x} \mathrm{e}^{j\left(t+\varphi_{x}\right)}\right\}$ is determined by the initial state. This harmonic oscillation is plugged into the equation of motion (6), of which the matching excitation

$$
\begin{aligned}
u(t)=\mathfrak{R e}\left\{2 D j \hat{x} \mathrm{e}^{j\left(t+\varphi_{x}\right)}+\varepsilon a_{2} \hat{x}^{2} \mathrm{e}^{2 j\left(t+\varphi_{x}\right)}\right. & \\
& \left.+\varepsilon a_{3} \hat{x}^{3} \mathrm{e}^{3 j\left(t+\varphi_{x}\right)}\right\}
\end{aligned}
$$

results. Note that the excitation frequency remains constant since the coefficients determining the eigenfrequency of the linear system do not change. For stability reasons, the controller does not update the excitation at every time step but after certain intervals, analogously to Section 3. As a consequence, the controller requires the parameters and the state variables only at these update times. To eliminate the disturbing influence of overshoots in the parameter estimation, their values are bounded with a critical rate of change. As long as the rate of change exceeds the critical rate, the system's excitation goes on with the latest admissible, estimated parameters of previous time steps.

\subsection{Parameter Estimation}

The parameter estimation differs significantly from that of the linear system since both transforms, Laplace- and Z-transform, are too involved for nonlinear systems. One way to obtain estimates for the parameters in the modified Duffing equation (6) is to estimate $\dot{x}$ and $\ddot{x}$ via finite differences. However, this is prone to measurement errors or noise [16]. Instead, we propose to recast (6) in a variational form. More precisely, we multiply (6) with a weighting function $w$ and integrate over the time interval $\left[t_{k-M+1}, t_{k}\right][3]$, which is called the measurement time $t_{\text {meas }}$. Hereby, $M$ is the number of time points per measurement. This yields

$$
\begin{aligned}
& \int_{t_{k-M+1}}^{t_{k}} w(t) \ddot{x}(t)+w(t) 2 D \dot{x}(t)+w(t) x(t) \\
& \quad+w(t) \varepsilon\left(a_{2} x^{2}(t)+a_{3} x^{3}(t)\right) \mathrm{d} t=\int_{t_{k-M+1}}^{t_{k}} w(t) u(t) \mathrm{d} t,
\end{aligned}
$$

which is referred to as measurement point. The measurement time is an algorithmic parameter and should be of the same order of magnitude as the period of the excited vibrations. The weighting function $w$ is assumed to be sufficiently smooth and to satisfy

$$
\begin{aligned}
& w\left(t_{k-M+1}\right)=w\left(t_{k}\right)=0, \\
& \dot{w}\left(t_{k-M+1}\right)=\dot{w}\left(t_{k}\right)=0 .
\end{aligned}
$$


Since we assume the position as sensor signal, the time derivatives of $x$ are shifted into the weighting function via integration by parts twice, resulting in

$$
\begin{aligned}
& \int_{t_{k-M+1}}^{t_{k}} \ddot{w}(t) x(t)-\dot{w}(t) 2 D x(t)+w(t) x(t) \\
& \quad+w(t) \varepsilon\left(a_{2} x^{2}(t)+a_{3} x^{3}(t)\right) \mathrm{d} t=\int_{t_{k-M+1}}^{t_{k}} w(t) u(t) \mathrm{d} t,
\end{aligned}
$$

where we assumed the sought parameters $a_{2}$ and $a_{3}$ to be constant during the measurement time, as we did in Section 3.2. For the sake of presentation, we introduce the quantities

$$
\begin{array}{ll}
I_{1}:=\int_{t_{k-M+1}}^{t_{k}} \ddot{w}(t) x(t) \mathrm{d} t, \quad I_{2}:=\int_{t_{k-M+1}}^{t_{k}} \dot{w}(t) 2 D x(t) \mathrm{d} t, \\
I_{3}:=\int_{t_{k-M+1}}^{t_{k}} w(t) x(t) \mathrm{d} t, \quad I_{4}:=\int_{t_{k-M+1}}^{t_{k}} w(t) \varepsilon x^{2}(t) \mathrm{d} t, \\
I_{5}:=\int_{t_{k-M+1}}^{t_{k}} w(t) \varepsilon x^{3}(t) \mathrm{d} t, \quad I_{u}:=\int_{t_{k-M+1}}^{t_{k}} w(t) u(t) \mathrm{d} t,
\end{array}
$$

which can be computed numerically by appropriate quadrature rules, such that a measurement point (7) can be written as

$$
a_{2} I_{4}+a_{3} I_{5}=I_{\mathrm{u}}-I_{1}+I_{2}-I_{3} .
$$

With a single equation only one parameter can be identified, so we accumulate $n$ measurement points

$$
\left[\begin{array}{cc}
I_{1,4} & I_{1,5} \\
I_{2,4} & I_{2,5} \\
\vdots & \vdots \\
I_{n, 4} & I_{n, 4}
\end{array}\right]\left[\begin{array}{l}
\tilde{a}_{2} \\
\tilde{a}_{3}
\end{array}\right]=\left[\begin{array}{c}
I_{1, \mathrm{u}}-I_{1,1}+I_{1,2}-I_{1,3} \\
I_{2, \mathrm{u}}-I_{2,1}+I_{2,2}-I_{2,3} \\
\vdots \\
I_{n, \mathrm{u}}-I_{n, 1}+I_{n, 2}-I_{n, 3}
\end{array}\right],
$$

where the first index $i=1, \ldots, n$ represents the use of different, preferably orthogonal, weighting functions $w_{i}$ or by including more of the time history, i.e., evaluations at previous time points $t_{k+1-i}$ or a combination of both. Since it is advantageous to set up more equations than unknowns, the Moore-Penrose pseudo-inverse is used to find a solution in the sense of least squares. To weight the latest measurement points the most, a fading memory is implemented as discussed in Section 3.2. Fast fluctuations (overshoots) in the parameter estimations are smoothed by a low-pass Chebyshev filter [14]. Note that the parameter estimation algorithm is not limited to weak nonlinearities, see the forthcoming section Section 5.2.2 for more details.

\subsection{State Observer}

Both velocity and position, have to be known by the controller in order to adapt the excitation to a time-varying system. The position is sensed, the velocity is not and needs to be reconstructed. Therefore a high gain state observer [1] is used. The real system is given by equation (6), whereas the observer system is described by

$$
\begin{aligned}
\dot{\tilde{x}}(t)= & \tilde{v}(t)+s_{1}(x(t)-\tilde{x}(t)), \\
\dot{\tilde{v}}(t)= & u(t)-2 D \tilde{v}(t)-\tilde{x}(t) \\
& -\varepsilon\left(a_{2} \tilde{x}^{2}(t)+a_{3} \tilde{x}^{3}(t)\right)+s_{2}(x(t)-\tilde{x}(t)) .
\end{aligned}
$$

For weak nonlinearity the linear terms dominate the dynamics of the observer error, thus the observer dynamics are tuned analogously to the Luenberger-Observer in Section 3.3.

\subsection{Example}

A rigorous analysis of the control loop for the nonlinear case including all interactions would be even more involved than in the linear case. Consequently, the performance of the proposed method is again assessed by numerical simulations. Estimated are the nonlinear parameters $a_{2}$ and $a_{3}$ of the modified Duffing equation (6).

The presented example is taken from tactile tissue measurements [20], which were adapted for diagnostics of skin. Its model and simulation parameters are presented in Table 2. As for the linear system in Section 3 , the controlled system is tested concerning its dynamic behavior and accuracy of the parameters to be measured.

The method is tested with the following step changes in the parameters:

$$
\begin{aligned}
& a_{2}(t)=2\left(1+\frac{1}{2} \cdot \operatorname{sign}\left(t-\frac{1}{3} t_{\text {sim }}\right)\right), \\
& a_{3}(t)=3\left(1+\frac{1}{2} \cdot \operatorname{sign}\left(t-\frac{2}{5} t_{\text {sim }}\right)\right) .
\end{aligned}
$$

At first the parameter $a_{2}$ is constant at 1.0 and changes at $t=\frac{1}{3} t_{\text {sim }}$ to the constant value of 3.0. The parameter $a_{3}$ is constant at 1.5 until $t=\frac{2}{3} t_{\text {sim }}$, where it takes the constant value 4.5. Such sudden parameter changes are usually a challenging problem for estimation algorithms.

As the integration is done in the limits $\left[t_{k-M+1}, t_{k}\right]$, with the sampling time of $\Delta t$ we obtain the measurement time $t_{\text {meas }}=M \Delta t$. We, therefore, choose the weighting function to be

$$
\begin{aligned}
& w_{1}(t)=\cos \left(2 \pi \frac{t-t_{k}}{M \Delta t}\right)-1, \\
& w_{2}(t)=\cos \left(4 \pi \frac{t-t_{k}}{M \Delta t}\right)-1 .
\end{aligned}
$$


Table 2 - Model (nondimensional) and simulation parameters of the modified Duffing oscillator

\begin{tabular}{llllll}
\hline variable description & variable & value & variable description & variable & value \\
\hline initial position & $x_{0}$ & 1 & simulation time & $t_{\text {sim }}$ & 100.5 \\
initial velocity & $\dot{x}_{0}$ & 0 & time step & $\Delta t$ & 0.042 \\
undamped radian eigenfrequency & $\omega_{0}$ & 1 & fading memory factor & $r$ & 0.8 \\
damping ratio & $D$ & 0.1 & time points per measurement & $M$ & 200 \\
frequency ratio & $\eta$ & 1 & total time steps & $N$ & 2400 \\
small parameter & $\varepsilon$ & $1 / 100$ & previous measurements included & $N_{\text {rts }}$ & 2 \\
quadratic coefficient & $a_{2}$ & $1.0-3.0$ & excitation update interval & $N_{\text {exc }}$ & 100 \\
cubic coefficient & $a_{3}$ & $1.5-4.5$ & observer dynamics & $\delta_{\text {obs }}$ & $10 D \omega_{0}$ \\
\hline
\end{tabular}

Strictly speaking depend these functions on two variable arguments $t$ and $t_{k}$ and should be denoted $w_{i}\left(t, t_{k}\right)$, for brevity of notation however, we omit the dependency on the current time point $t_{k}$. It is easy to see that the weighting functions $w_{1}$ and $w_{2}$ satisfy the conditions in (8). The results in Figure 7 and Figure 8 show how close the estimated parameters follow the actual ones. At the beginning of the simulation, the estimated parameters are initialized with zero. The need for an initial phase is caused by the necessity to collect enough measurement points. The change of $a_{2}$ at $t=\frac{1}{3} t_{\text {sim }}$ (of $a_{3}$ at $t=\frac{2}{3} t_{\text {sim }}$, respectively) follows a smooth shift of the estimated parameter $\tilde{a}_{2}$ ( $\tilde{a}_{3}$ respectively). During the transition time, until most sensed data belong to dynamics with the new parameter values, the parameter estimations overshoot and approach close to the actual values. The smooth transition without large spikes is attributed to the overdetermined system of equations, using four equations (two weighting functions $w_{1}$ and $w_{2}$, over two time intervals $\left[t_{k-M}, t_{k-1}\right]$ and $\left.\left[t_{k-M+1}, t_{k}\right]\right)$ than unknowns - namely the parameters $a_{2}$ and $a_{3}$ and the low-pass filtering. After less than a measurement time $t_{\text {meas }}$, i.e., when still time points belonging to the old parameter value enter the calculation, the estimated parameters come close to the real values. The persistent deviation after the change is less than $1 \%$ for the parameter $a_{2}$ and less than $3 \%$ for the parameter $a_{3}$. There are cross-couplings between $a_{2}$ and $a_{3}$ resulting in temporary deviations of about $20 \%$, meaning that changes in one parameter also affect the estimation of the other.

Furthermore, a very close adaption of the velocity can be obtained (cf. Figure 9) with the presented state observer.

The low-pass filtered parameter estimations lead to a stable and smooth excitation. As presented in Figure 10, the system response is harmonic as desired and has large, well detectable amplitudes. There are slight fluctuations in the excitation since it is based on the observed state and the estimated parameters. Also, the excitation $u$ deviates qualitatively from the response $x$

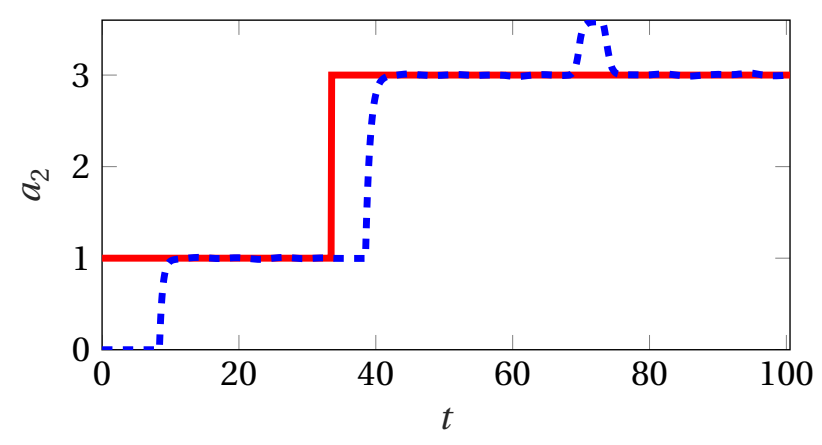

Figure 7 - Real (solid red line) and estimated (dashed blue line) system parameter $a_{2}$

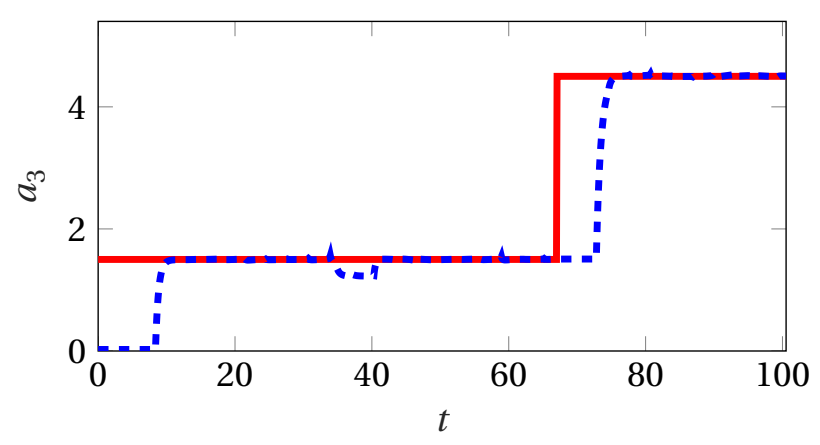

Figure 8 - Real (solid red line) and estimated (dashed blue line) system parameter $a_{3}$

when the estimated parameters $a_{2}$ and $a_{3}$ increase, as the nonlinear terms gain a higher impact.

A popular alternative for estimating parameters is the extended Kalman filter. We prefer our method over the Kalman filter, because the Kalman filter is related to convergence issues, whereas our method returns parameter estimations in a single step. After the initial phase, there are enough data to calculate new parameter estimates algebraically. Furthermore, the computational cost is lower, since only the weighting functions and their derivatives have to be provided which can readily be precalculated and stored. Consequently, the typical drawbacks of iterative methods like the convergence speed or radius as well as the recalculation of the weighting matrices are avoided. 


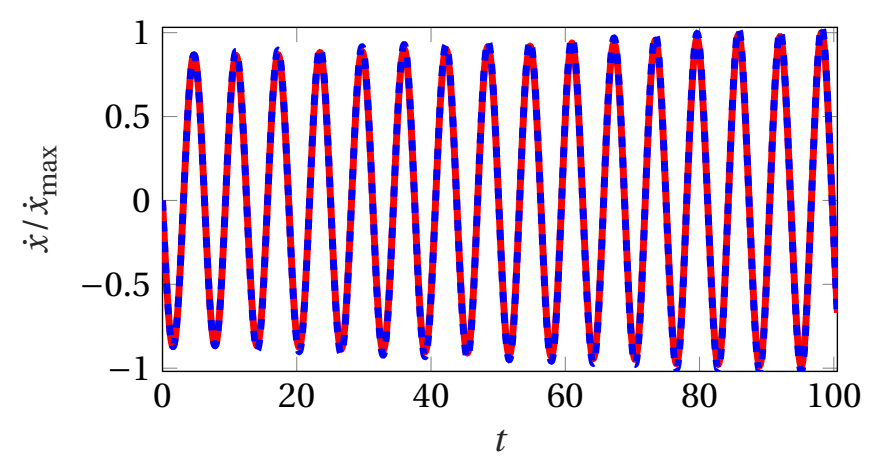

Figure 9-Real (solid red line) and observed (dashed blue line) velocity of the modified Duffing oscillator

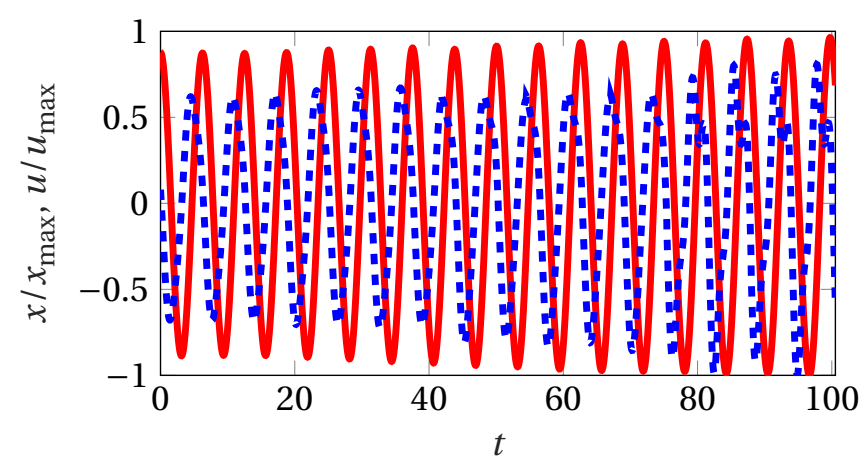

Figure 10 - Position $x$ (solid red line) and excitation $u$ (dashed blue line) of the modified Duffing oscillator

\section{Discussion}

The proposed approach proved to measure continuously three parameters of a linear oscillator and two parameters of a weakly nonlinear oscillator. Naturally, there arise questions for the estimation of more parameters at a time or the extensions to further system classes. We address some of these questions in the following subsections.

\subsection{Limitations}

Both approaches, for linear systems via Z-transform as well as for nonlinear systems via weighting functions, may lead to ill-conditioned systems of equations and thus result in corrupted parameter estimates. The underlying problems are similar for both approaches. In the following, we discuss the details for the weighting function approach. If the excitation is a harmonic function, the corresponding steady-state response may cause linearly dependent columns in the linear system of equations for the parameters. A simple example can explain this. For a damped oscillator, the solution of the homogeneous equation decays, while the stationary response to a harmonic excitation reads

$$
x_{p}(t)=\hat{x} \cos \left(\Omega t+\varphi_{x}\right), \quad \ddot{x}_{p}(t)=-\hat{x} \Omega^{2} \cos \left(\Omega t+\varphi_{x}\right) .
$$

The crucial point is the second derivative $\ddot{x}_{p}$ and possibly higher derivatives $x_{p}^{(2 k)}$ of even parity. Similarly, the first derivative $\dot{x}_{p}$ and possibly higher derivatives $x_{p}^{(2 k+1)}$ of odd parity may cause that problem as well. These terms differ only by a constant factor. More precisely let us index the measurement points with $i=1,2, \ldots, n$ with $i=1$ denoting the newest and $i=n$ the oldest measurement point. For example, we have

$$
I_{i, 3}=\int_{t_{k+1-i-M}}^{t_{k+1-i}} w(t) x(t) \mathrm{d} t \quad \text { for } i=1,2, \ldots, n,
$$

and similarly for the remaining integrals in equation (9). Then the linear system we have to solve is of the form

$$
\left[\begin{array}{ccc}
-\Omega^{2} I_{1,3} & I_{1,2} & I_{1,3} \\
-\Omega^{2} I_{2,3} & I_{2,2} & I_{2,3} \\
\vdots & \vdots & \vdots \\
-\Omega^{2} I_{n, 3} & I_{n, 2} & I_{n, 3}
\end{array}\right]\left[\begin{array}{c}
\tilde{m} \\
\tilde{d} \\
\tilde{c}
\end{array}\right]=\left[\begin{array}{c}
I_{1, \mathrm{u}} \\
I_{2, \mathrm{u}} \\
\vdots \\
I_{n, \mathrm{u}}
\end{array}\right] .
$$

The first and third columns of the coefficient matrix are linearly dependent in stationary state. The reason is evident when looking at these coefficients before integration by parts. The first column contains integrals $\int \ddot{x}(t) w(t) \mathrm{d} t$ and the third column $\int x(t) w(t) \mathrm{d} t$. As a consequence, the coefficient matrix becomes rank deficient after a certain time, and the number of parameters that remains identifiable is only two, either $m$ and $d$, or $d$ and $c$. This observation is in agreement with a general condition for parameter convergence with persistent excitation [4], stating roughly that two parameters can be estimated per frequency in the excitation signal. During transient oscillations, the linear system of equations for more than two parameters is well-conditioned and gives accurate estimations. This is due to the transient system responses changing the values of the derivatives. One may have expected that parameter estimations improve as the signal converges to its stationary solution, but this is not the case.

Similarly, one may not be able to distinguish the zero solution from potential other solutions, if the right-hand side vanishes. Since the weighting functions are periodic by condition (8), the product of them with a periodic excitation with vanishing mean value is periodic or at least quasiperiodic. Consequently, the integral $I_{u}=F\left(t_{k}\right)-F\left(t_{k-M+1}\right)$, with $F(t)=\int_{0}^{t} w(\tau) u(\tau) \mathrm{d} \tau$, vanishes, either due to the measurement time $t_{\text {meas }}$ or due to the current time. Former happens when the measurement time equals a multiple of the period of $F(t)$, then 
for all time points $F\left(t_{k}\right)=F\left(t_{k-M+1}\right)$ and thus $I_{u}=0$ A similar effect occurs when the measurement time is much longer than the period of the excited vibrations. For other measurement times, there is always a time point of a continuous periodic function for which $F\left(t_{k}\right)=F\left(t_{k-M+1}\right)$, leading to spikes in the parameter estimation, if additional measurement points do not overdetermine the system of equations.

A problem in experiments occurs when the sensor signal has an offset because numerical integration is very sensitive to offset errors. Sensor noise evens out by the integration, but a sensor offset grows with time. However, this can be resolved by introducing an additional parameter $p_{\mathrm{O}}$. This parameter can be interpreted as the factor of a constant function $x_{\mathrm{O}}(t)=1$. The corresponding integral $\int p_{\mathrm{O}} w(t) x_{\mathrm{O}}(t) \mathrm{d} t$ adds a fourth column to the linear system of equations for the parameter estimates

$$
\left[\begin{array}{cccc}
I_{1,1} & I_{1,2} & I_{1,3} & I_{1,4} \\
I_{2,1} & I_{2,2} & I_{2,3} & I_{2,4} \\
\vdots & \vdots & \vdots & \vdots \\
I_{n, 1} & I_{n, 2} & I_{n, 3} & I_{n, 4}
\end{array}\right]\left[\begin{array}{c}
\tilde{m} \\
\tilde{d} \\
\tilde{c} \\
\tilde{p}
\end{array}\right]=\left[\begin{array}{c}
I_{1, \mathrm{u}} \\
I_{2, \mathrm{u}} \\
\vdots \\
I_{n, \mathrm{u}}
\end{array}\right] .
$$

As a result, not only the parameters of the system but also the offset $p_{\mathrm{O}}$ is obtained.

\subsection{Generalizations}

The extension of both approaches to SISO systems of higher order is straightforward. For linear systems, there are just more coefficients in the Laplace- and Z-transform, while for weakly nonlinear systems, we need to formulate further requirements on the weighting functions depending on the number of differentiations or integrations needed to derive an algebraic equation.

We will outline the generalizations of the parameter estimation methods to linear MIMO systems and nonlinear SISO systems in the following subsections. Although the approach is not limited to oscillatory systems, we restrict ourselves to oscillatory systems, since we believe this is the most relevant class for technical applications.

\subsubsection{Linear MIMO Systems}

The introduced control concept can be applied to linear oscillatory systems with many degrees of freedom. For this kind of system, it is convenient to switch to state space form. The state vector $\mathbf{x}(t) \in \mathbb{R}^{n}$, the input vector $\mathbf{u}(t) \in \mathbb{R}^{m}$ and the output vector $\mathbf{y}(t) \in \mathbb{R}^{k}$ are related by the set of linear equations

$$
\begin{aligned}
\dot{\mathbf{x}}(t) & =\mathbf{A}(t) \mathbf{x}(t)+\mathbf{B}(t) \mathbf{u}(t), \\
\mathbf{y}(t) & =\mathbf{C}(t) \mathbf{x}(t) .
\end{aligned}
$$

with matrices $\mathbf{A} \in \mathbb{R}^{n \times n}, \mathbf{B} \in \mathbb{R}^{n \times m}$, and $\mathbf{C} \in \mathbb{R}^{k \times n}$ and $t \in \mathbb{R}$ the time. Assuming a zero initial condition and applying the Laplace transform to equation (10) and rearranging the terms yields the so-called transfer function

$$
G(s)=\mathbf{C}(s \mathbf{I}-\mathbf{A})^{-1} \mathbf{B} .
$$

The system of equations (10) is called oscillatory if there exists a complex conjugated pair of poles of $G$. It is called asymptotically stable if the maximal real part of all poles of $G$ is less than zero.

As in the previous sections, we assume that the linear system (10) is sampled at discrete time points and thus, we consider the discrete-time counterpart of (10), given by

$$
\begin{aligned}
\mathbf{x}\left(t_{k}+1\right) & =\mathbf{A}_{Z}\left(t_{k}\right) \mathbf{x}\left(t_{k}\right)+\mathbf{B}_{Z}\left(t_{k}\right) \mathbf{u}\left(t_{k}\right), \\
\mathbf{y}\left(t_{k}\right) & =\mathbf{C}_{Z}\left(t_{k}\right) \mathbf{x}\left(t_{k}\right) .
\end{aligned}
$$

The challenging part is to express the discrete-time matrices $\mathbf{A}_{Z}, \mathbf{B}_{Z}$, and $\mathbf{C}_{Z}$ such that all known information of the continuous-time matrices $\mathbf{A}, \mathbf{B}$, and $\mathbf{C}$ enter them and the unknown entries of $\mathbf{A}, \mathbf{B}$, and $\mathbf{C}$ can be recovered from as little time points $t_{k}, \ldots, t_{k-M}$ as possible.

For multiple inputs it is reasonable to fit them to an eigenmode, corresponding to the eigenfrequency which is to be excited.

Note that the simultaneous excitation of several eigenfrequencies may have beneficial effects, e.g., for the cancellation of temperature effects in fluid characterization [5].

\subsubsection{Nonlinear Systems}

The approach is limited to combinations of system models and sensor signals, where all integrals of the measurement points can be integrated by parts analytically.

E.g., this approach still works for measuring the parameter $\alpha$ of a classical Van-der-Pol oscillator

$$
\begin{aligned}
\ddot{x}(t)-\alpha\left(1-x^{2}(t)\right) \dot{x}(t)+x(t) & =u(t), \\
x\left(t_{0}\right) & =x_{0}, \\
\dot{x}\left(t_{0}\right) & =\dot{x}_{0},
\end{aligned}
$$

when only the time history $x(t)$ is available, because the nonlinear term

$$
\int x(t)^{2} \dot{x}(t) w(t) \mathrm{d} t=-\int \frac{1}{3} x(t)^{3} \dot{w}(t) \mathrm{d} t
$$

is easy to integrate. However, for general integrals, e.g., $\int x(t)^{2} \dot{x}(t)^{2} \mathrm{~d} t$, it may be difficult to find a representation without $\dot{x}(t)$. 
A nonlinear, time-dependent MIMO system in statespace form is described by

$$
\begin{aligned}
& \dot{\mathbf{x}}(t)=\mathbf{f}\left(\mathbf{x}(t), \mathbf{u}(t), \mathbf{p}_{1}(t), t\right), \quad \mathbf{x}\left(t_{0}\right)=\mathbf{x}_{0}, \\
& \mathbf{y}(t)=\mathbf{c}\left(\mathbf{x}(t), \mathbf{p}_{2}(t), t\right) .
\end{aligned}
$$

The parameters of the system and the output equation are collected in the vectors $\mathbf{p}_{1}(t) \in \mathbb{R}^{\ell_{1}}$ and $\mathbf{p}_{2}(t) \in \mathbb{R}^{\ell_{2}}$, respectively. This system class is too complicated for uniform treatment. Even the basic property oscillatory, which is essential for vibration-based measurements, could be defined only via Poincare maps of the free dynamics $(\mathbf{u}=\mathbf{0})$. The requirements are similar to a nonlinear SISO system, i.e., the unsensed states should not appear in the integrals of the measurement points, but the multiple inputs and outputs may cause additional nonlinear coupling terms. This makes the generalization to this system class feasible, but it needs to be decided on a case-by-case basis.

\section{Conclusion and Outlook}

The combination of adaptive control with resonance tracking is a promising approach to measure several parameters simultaneously and continuously in a vibrating system. Simultaneous measurement of parameters would be challenging with conventional methods, such as autoresonance and phase control. The proposed control design for resonance tracking allows to track sudden parameter changes of more than $100 \%$ by a settling time of a few oscillation periods, including cross-coupling effects, i.e., changes in one parameter affect the estimation of unchanged parameters. The measurement of slow changes exhibits the same accuracy as for constant values and can be reduced to arbitrary precision. An interesting observation is the high measurement performance, particularly at transient oscillations.

However, some of the design goals of the closed loop conflict: it is not possible to obtain high dynamics and a low steady-state error at the same time. The critical point is the number of tuning parameters, such as measurement time, controller update time, parameters of the low-pass filter, fading memory, observer dynamics, even for a different choice of parameter estimators and state observers. On the one hand, they may be chosen to obtain high accuracy and fast dynamics, on the other hand, they may destabilize the system. Further research for guiding or ideally automating the adjustment of the estimator, observer, and controller is necessary.

If instead of an oscillation with one frequency, a multifrequent oscillation would be acceptable in the application at hand, the control loop would dispense with the state observer. In this case, the input signal may be constructed as a signal of constant amplitude with timevarying frequency according to the estimated system parameters. Moreover, also multifrequent excitation signals are worth for consideration of further improvements of the measurement system. In a more theoretical perspective, the limitations of the proposed parameter estimation should be analyzed in-depth. The parameters are estimated at each time point in a single step. Although this is the strength of this approach, it also poses a weakness, since the system may become illconditioned, if the excitation and the system response are periodic. However, in comparison to the Kalmanfilter, the proposed method is indeed a promising approach, as for the obligatory initialization step the weighting function is calculated once, rather than updating the Kalman-matrix at each step.

Code Availability: We provide commented MATLAB files of a demonstration example as supplementary material, which can be found under the

DOI: 10.14464/gammas.vli1.344.

\section{References}

[1] J. Adamy. Nichtlineare Regelungen. Springer-Verlag, 2009. ISBN 978-3-642-00794-1.

[2] V.K. Astashev and V.I. Babitsky. Ultrasonic processes and machines: dynamics, control and applications. Springer Science \& Business Media, 2007. ISBN 978-3-540-72061-4.

[3] U. Beckert and H. Arnold. Identification and verification of parameters of the asynchronous machine during field-orientedly controlled operation. In International Congress on Electrical Machines (ICEM 2004), 2004. Proc. 16. Int. Conf. Electr. Mach. Krakau (2004), p. 1005-1007.

[4] S. Boyd and S.S. Sastry. Necessary and sufficient conditions for parameter convergence in adaptive control. Automatica, 22(6): 629-639, 1986.

[5] T. Brack. Multi-Frequency Phase Control of a Torsional Oscillator for Applications in Dynamic Fluid Sensing (Diss.No. 23952). PhD thesis, ETH Zurich, 2017.

[6] T. Brack, D. Kern, M. Chen, and J. Dual. Dynamics and stability of phase controlled oscillators. Journal of Dynamic Systems, Measurement, and Control, 138(7):071007, 2016.

[7] C. Gokcek. Tracking the resonance frequency of a series RLC circuit using a phase locked loop. In Control Applications, 2003. CCA 2003. Proceedings of 2003 IEEE Conference on, pages 609-613, 2003.

[8] J.W. Grate, S.J. Martin, and R.M. White. Acoustic wave microsensors. Analytical Chemistry, 65(21):940A-948A, 1993.

[9] T. Hemsel, R. Stroop, D.O. Uribe, and J. Wallaschek. Resonant vibrating sensors for tactile tissue differentiation. Journal of Sound and Vibration, 308(3):441 - 446, 2007. ISSN 0022-460X.

[10] I.D. Landau, R. Lozano, M. M'Saad, and A. Karimi. Adaptive control, volume 51. Springer Berlin, 1998. ISBN 978-0-85729664-1.

[11] D. Luenberger. Observers for multivariable systems. IEEE Transactions on Automatic Control, 11(2):190-197, 1966.

[12] S. Priya and D.J. Inman. Energy harvesting technologies, volume 21. Springer, 2009. ISBN 978-0-387-76464-1. 
[13] D. Scheibner, J. Wibbeler, J. Mehner, B. Brämer, T. Gessner, and W. Dötzel. A frequency selective silicon vibration sensor with direct electrostatic stiffness modulation. Analog Integrated Circuits and Signal Processing, 37(1):35-43, 2003.

[14] I.W. Selesnick and C.S. Burrus. Generalized digital butterworth filter design. IEEE Transactions on Signal Processing, 46(6): 1688-1694, 1998.

[15] A.M. Shkel, R. Horowitz, A. Seshia, S. Park, and R.T. Howe. Dynamics and control of micromachined gyroscopes. In Proceedings of the 1999 American Control Conference (Cat. No. 99CH36251), volume 3, pages 2119-2124. IEEE, 1999.

[16] F. Thuselt and F.P. Gennrich. Numerische Integration und Differentiation. In Praktische Mathematik mit MATLAB, Scilab und Octave, pages 323-360. Springer, 2013.

[17] A. Tustin. A method of analysing the behaviour of linear systems in terms of time series. Journal of the Institution of Electrical Engineers-Part IIA: Automatic Regulators and Servo Mechanisms, 94(1):130-142, 1947.

[18] J. Twiefel, M. Klubal, C. Paiz, S. Mojrzisch, and H. Krüger. Digital signal processing for an adaptive phase-locked loop controller. In Modeling, Signal Processing and Control for Smart Structures 2008, volume 6926, pages 6926-6926-12, 2008.

[19] H. Unbehauen. Regelungstechnik, Band 2: Zustandsregelungen, digitale und nichtlineare Regelsysteme, 9. Auflage. Vieweg Verlag, Braunschweig, 2000. ISBN 978-3-528-83348-0.

[20] D.O. Uribe, R. Stroop, T. Hemsel, and J. Wallaschek. Development of a biomedical tissue differentiation system using piezoelectric actuators. In Frequency Control Symposium, 2008 IEEE International, pages 91-94. IEEE, 2008.

[21] S. Voronina and V. Babitsky. Autoresonant control strategies of loaded ultrasonic transducer for machining applications. Journal of Sound and Vibration, 313(3):395-417, 2008.

[22] M.J. Wadas, M. Tweardy, N. Bajaj, A.K. Murray, G.T.C. Chiu, E.A. Nauman, and J.F. Rhoads. Detection of traumatic brain injury protein biomarkers with resonant microsystems. IEEE Sensors Letters, 1(6):1-4, 2017.

[23] C. Wagner, M.F.B. Green, P. Leinen, T. Deilmann, P. Krüger, M. Rohlfing, R. Temirov, and F.S. Tautz. Scanning quantum dot microscopy. Phys. Rev. Lett., 115:026101, Jul 2015. 